\title{
Method of Selecting a Radar Sample Preferably
}

\author{
Andrey A. Tsyganov* \\ Military Academy of Aero-Space Defense \\ named after the Marshal of Soviet Union G.K. Zhukov \\ 50 Zhigareva Str., Tver, 170100, Russia
}

Received 16.10.2018, received in revised form 10.01.2019, accepted 23.04.2019

A new method of selecting a radar sample preferably has been developed. The methods is based on the representation in the form of an additive function of the estimated functional of each normalized indicator characterizing the radar. For each variant of the compared samples, which makes it possible to evaluate the effectiveness of the radar with the help of a single integral indicator, and also to eliminate the uncertainty in the choice of the radar by taking into account additional indicators and to take into account the cost radar to the maximum of the preference function.

Keywords: selecting a radar, indicator.

Citation: Tsyganov A.A. Method of selecting a radar sample preferably, J. Sib. Fed. Univ. Eng. technol., 2019, 12(6), 666-672. DOI: $10.17516 / 1999-494 X-0165$.

\section{Метод выбора предпочтительного образца радиолокационной станции}

\author{
А.А. Цыганов \\ Военная академия воздушно-космической оборонь \\ им. Маршала Советского Союза Г.К. Жукова \\ Россия, 170100, Тверь, ул. Жигарева, 50
}

\begin{abstract}
Разработан новый метод выбора образияа радиолокаџионной станции. Метод основан на представлении в виде аддитивной функции оценочных функиионалов каждого нормированного показателя, характеризующего радиолокачионную станцию, для каждого варианта сравниваемых образиов, что дает возможность оченивать эффективность РЛС с помощью одного интегрального показателя, исключить неопределенность при выборе конкретных типов РЛС за счет учета дополнительных показателей, учитывать стоимость РЛС по максимуму функции предпочтения.
\end{abstract}

(C) Siberian Federal University. All rights reserved

This work is licensed under a Creative Commons Attribution-NonCommercial 4.0 International License (CC BY-NC 4.0).

* Corresponding author E-mail address: kuchin.a.a@gmail.com 
Любой образец радиолокационной станции (РЛС) как сложный объект характеризуется множеством частных показателей - тактико-технико-экономических характеристик (ТТЭХ), которые могут быть сведены в определенные группы, такие как интегральный показатель «Эффективность РЛС», комплексные показатели «Информационные возможности», «Живучесть», «Эксплуатационные характеристики», «Мобильность» и «Стоимость». Общая иерархическая структура частных показателей качества РЛС включает более 100 ТТЭХ. При этом все ТТЭХ сводятся в группы показателей, причем в каждую из групп входит от 3 до 10 показателей, т. е. процесс выбора наиболее предпочтительного варианта образца РЛС будет зависеть от множества тактических, технических и стоимостных характеристик образцов, что в свою очередь у лица, принимающего решение (ЛПР), может вызывать при выборе затруднения.

Необходимость обоснования выбора того или иного типа радиолокационной станции у лица, принимающего решение, возникает либо при необходимости повышения эффективности группировки из-за усложнения прогнозируемых целевой и помеховой обстановки по причине изменения состава СВКН противника и варианта ударов, либо при переоснащении группировки на новые типы образцов РЛС с более высокими требованиями к ТТХ, а также при создании новых группировок в районах, где они отсутствовали.

В общем случае процесс выбора РЛС основывается на оценке степени соответствия возможностей образца требованиям потребителей радиолокационной информации (РЛИ). При этом выбор РЛС проводится следующим способом: представление интегрального показателя «Эффективность» РЛС в виде свертки комплексных показателей качества РЛС с использованием весовых коэффициентов, определяющих «вклад в конечную эффективность» каждого из них. При этом комплексные показатели качества РЛС также получают методом свертки, но уже частных показателей (тактико-технических характеристик) станций с соответствующими весами. Данный вариант является наиболее предпочтительным (и чаще всего практически реализуемым) в условиях дефицита исходных данных или в условиях временных ограничений на проведение оценок.

Представление показателей качества РЛС в виде иерархической системы с рассмотрением на каждом ее уровне небольшого числа одноплановых показателей в каждой группе, отбор наиболее информативных из них облегчают работу привлекаемых экспертов и повышают объективность экспертных оценок важности тех или иных свойств и аспектов сравниваемых альтернатив. В связи с тем, что возможности РЛС характеризуются несколькими показателями, задача выбора предпочтительного образца РЛС сводится к решению многокритериальной задачи, что представляет определенную сложность и требует значительных вычислительных ресурсов и времени. В этих условиях необходим поиск решения, направленного на действительно эффективное решение задачи выбора образца РЛС.

Предлагаемый метод включает в себя новые, ранее не оцениваемые, частные показатели такие, как:

- вероятность разрешения воздушных объектов (BО) в группе РЛС различного диапазона волн при заданных параметрах БП противника [1];

$$
-667-
$$


- вероятность распознавания классов (типов) воздушных объектов (ВО) по сигнальным признакам [2];

- коэффициент наблюдаемости при проводке ВО в условиях пассивных помех (ПП) [3];

- стоимость эксплуатации РЛС в течение срока службы $[4,5]$.

Вышеперечисленные показатели позволят исключить неопределенность при выборе конкретных типов РЛС.

Под предпочтительным образцом радиолокационной станции понимается такой образец, стоимость которого будет не выше требуемой, а эффективность будет максимальной среди сравниваемых.

Для каждого показателя, характеризующего радиолокационную станцию, с помощью метода парных сравнений определяется коэффициент значимости с учетом предназначения радиолокационной станции (радиолокационная станция малых высот и радиолокационная станция средних и больших высот и т.д.). С учетом указанных коэффициентов значимости формируется функция предпочтения в виде аддитивной свертки.

Из всего множества сравниваемых образцов выбираются те образцы, стоимость которых не выше заданной, и далее из отобранных формируется множество радиолокационных станций, удовлетворяющих требованиям по стоимости.

Из полученного множества радиолокационных станций, удовлетворяющих требованиям по стоимости, выбирается образец с максимальным значением функции предпочтения, который и будет являться предпочтительным.

Для каждой радиолокационной станции формируется таблица, в которую заносятся значения показателей, характеризующих возможности радиолокационной станции (табл. 1).

Эксперты определяют весовые коэффициенты каждого из вышеперечисленных показателей. Весовые коэффициенты выбираются экспертами исходя из требуемого варианта при-

Таблица 1. Показатели, характеризующие возможности радиолокационной станции

Table 1. Indicators, characterizing the radar capabilities

\begin{tabular}{|c|l|l|}
\hline № показателя & Обозначение показателя & \multicolumn{1}{|c|}{$\begin{array}{c}\text { Показатель, характеризующий } \\
\text { радиолокационную станцию }\end{array}$} \\
\hline 1 & $k_{1}$ & Дальность обнаружения \\
\hline 2 & $k_{2}$ & Коэффициент подавления помех \\
\hline 3 & $k_{3}$ & Коэффициент подпомеховой видимости \\
\hline 4 & $k_{4}$ & Средняя наработка на отказ \\
\hline 5 & $k_{5}$ & Среднее время восстановления \\
\hline 6 & $k_{6}$ & Потребляемая мощность \\
\hline 7 & $k_{7}$ & Точность определения координат \\
\hline 8 & $k_{8}$ & Вероятность разрешения ВО в группе \\
\hline 9 & $k_{9}$ & Максимальная высота беспровальной проводки \\
\hline 10 & $k_{10}$ & Вероятность распознавания классов (типов) ВО \\
\hline 11 & $k_{11}$ & Коэффициент наблюдаемости \\
\hline
\end{tabular}


менения выбираемой радиолокационной станции (обнаружение и сопровождение воздушных объектов на малых высотах в беспомеховой обстановке, обнаружение и сопровождение воздушных объектов на средних и больших высотах в беспомеховой обстановке, обнаружение и сопровождение воздушных объектов на малых высотах в условиях воздействия помех, обнаружение и сопровождение воздушных объектов на средних и больших высотах в условиях воздействия помех).

Данная операция проводится каждым экспертом с использованием метода парных сравнений, который позволяет произвести поочередное сравнение двух показателей, игнорируя все остальные, что значительно облегчает процесс принятия решения.

Для этого список всех показателей заносится в таблицу парных сравнений, и далее каждый эксперт принимает решение о поочередной оценке значимости каждого показателя (значимость каждого показателя не должна превышать 1), при сравнении его с остальными, путем распределения своего голоса между двумя сравниваемыми показателям. И, например, если значимость сравниваемых двух показателей, по мнению эксперта, одинакова, то в таблицу заносятся числа 0,5 и 0,5 , или если значимость первого показателя, по мнению эксперта, значительно превосходит значимость второго показателя, то в таблицу заносятся числа 0,9 и 0,1 (табл. 2).

Далее формируется общая таблица парных сравнений всех экспертов, которая имеет такой же вид, что и таблица парных сравнений для одного эксперта, при этом значением $\left(r_{i j}^{*}\right)$ каждой ячейки данной таблицы является сумма значений $\left(r_{i j}\right)$ этой же ячейки в таблицах всех экспертов

$$
r_{i j}^{*}=\sum_{k=1}^{N} r_{i j k}
$$

Таблица 2. Таблица, заполняемая каждым экспертом

Table 2 . The table filled by each expert

\begin{tabular}{|c|c|c|c|c|c|c|c|c|c|c|c|}
\hline $\begin{array}{c}\text { Наименование } \\
\text { показателя }\end{array}$ & 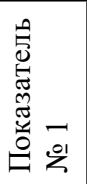 & 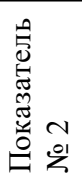 & 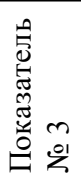 & 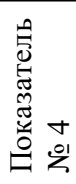 & 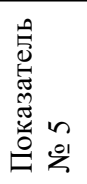 & 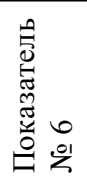 & 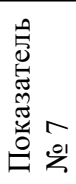 & 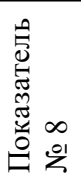 & 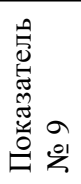 & 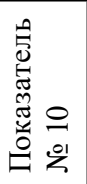 & 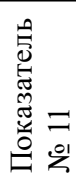 \\
\hline Показатель № 1 & - & 0,7 & 0,4 & 0,2 & 0 & 0,2 & 0,7 & 0,9 & 0,4 & 0,7 & 0,8 \\
\hline Показатель № 2 & 0,3 & - & 0,5 & 0,4 & 0,1 & 0,8 & 0,9 & 0,3 & 0,5 & 0,6 & 0,4 \\
\hline Показатель № 3 & 0,6 & 0,5 & - & 0,9 & 0,3 & 0,2 & 0,4 & 0,9 & 0,7 & 0,3 & 0,3 \\
\hline Показатель № 4 & 0,8 & 0,6 & 0,1 & - & 0,2 & 0,5 & 0,2 & 0,1 & 0,2 & 0,8 & 0,9 \\
\hline Показатель № 5 & 1 & 0,9 & 0,7 & 0,8 & - & 0,9 & 0,9 & 0,6 & 0,9 & 0,1 & 0,6 \\
\hline Показатель № 6 & 0,8 & 0,2 & 0,8 & 0,5 & 0,1 & - & 0,1 & 0,4 & 0,3 & 0,6 & 0,5 \\
\hline Показатель № 7 & 0,3 & 0,1 & 0,6 & 0,8 & 0,1 & 0,9 & - & 0,1 & 0,8 & 0,5 & 0,8 \\
\hline Показатель № 8 & 0,1 & 0,7 & 0,1 & 0,9 & 0,4 & 0,6 & 0,9 & - & 0,1 & 0,7 & 0,6 \\
\hline Показатель № 9 & 0,6 & 0,5 & 0,3 & 0,8 & 0,1 & 0,7 & 0,2 & 0,9 & - & 0,4 & 0,7 \\
\hline Показатель № 10 & 0,3 & 0,4 & 0,7 & 0,2 & 0,9 & 0,4 & 0,5 & 0,3 & 0,6 & - & 0,8 \\
\hline Показатель № 11 & 0,2 & 0,6 & 0,7 & 0,1 & 0,4 & 0,5 & 0,2 & 0,4 & 0,3 & 0,2 & - \\
\hline
\end{tabular}


где $N$ - число экспертов; $i=\overline{1,11} ; j=\overline{1,11} ; 11$ - число показателей, характеризующих возможности радиолокационной станции.

Далее производится определение суммарного уровня значимости $\left(R_{j}^{*}\right)$ для $j$-го показателя:

$$
R_{j}^{*}=\sum_{i=1}^{11} r_{i j}^{*}
$$

Полученные значения нормируются по формуле

$$
R_{j}=\frac{R_{j}^{*}}{\sum_{j=1}^{11} R_{j}^{*}}
$$

таким образом, чтобы выполнялось условие

$$
\sum_{j=1}^{11} R_{j}=1 .
$$

В табл. 3 показан пример общей таблицы парных сравнений.

Результатом проведения данного этапа является множество $(R)$ коэффициентов $\left(R_{i}\right)$ важности $i$-го показателя:

$$
R=\left\{R_{1}, R_{2}, \ldots, R_{9}\right\}
$$

Далее на основе значения показателей, характеризующих возможности радиолокационной станции, определяется коэффициент соответствия значений показателей требуемым значениям этих показателей (определяются заказчиком) [6]:

$$
q_{i} j=\frac{k_{i}^{*}}{k_{i} j},(i=\overline{1,11}),(j=\overline{1,11}),
$$

где $k_{i j}$ - значение $i$-го показателя, характеризующего $j$-ю станцию; $k_{i}^{*}$ - требуемое значение $i$-го показателя; $M$ - число радиолокационных станций, из которых производится выбор.

Далее для каждой радиолокационной станции определяется значение аддитивной функции предпочтения $W_{j}$ с учетом определенных коэффициентов важности из множества $R$ :

$$
W_{j}=\sum_{i=1}^{11}\left(R_{i j} q_{i j}\right),(j=\overline{1,11}),
$$

где $M$ - число радиолокационных станций, из которых производится выбор; $R_{i j}$ - значение коэффициента важности для $i$-го показателя, характеризующего $j$-ю радиолокационную станцию; $q_{i j}$ - значение $i$-го коэффициента соответствия.

Результатом проведения данного этапа будет являться множество значений функции предпочтения для каждой радиолокационной станции, из которых необходимо производить выбор:

$$
W=\left\{W_{1}, W_{2}, \ldots, W_{M}\right\}
$$

где $M$ - число радиолокационных станций, из которых необходимо производить выбор. 
Таблица 3. Таблица парных сравнений

Table 3 The table of paired comparations

\begin{tabular}{|c|c|c|c|c|c|c|c|c|c|c|c|c|c|}
\hline 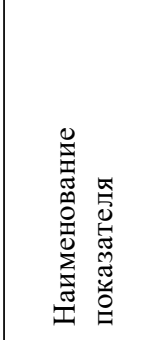 & 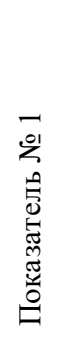 & 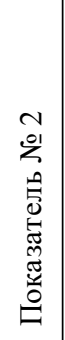 & 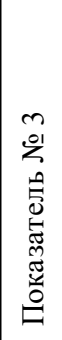 & 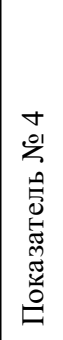 & 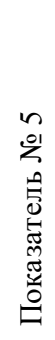 & 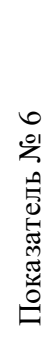 & 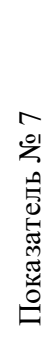 & 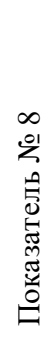 & 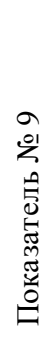 & 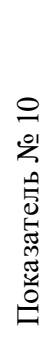 & 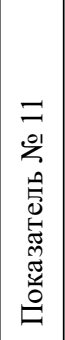 & $\begin{array}{c}\text { Суммарный уровень } \\
\text { значимости j-го } \\
\text { показателя }\left(\mathrm{R}^{*} \mathrm{j}\right)\end{array}$ & 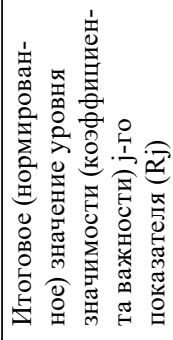 \\
\hline $\begin{array}{l}\text { Показа- } \\
\text { тель № } 1\end{array}$ & - & 2,3 & 2,0 & 1,2 & 1,9 & 1,8 & 1,2 & 1,7 & 2,2 & 2,5 & 1,8 & $\begin{array}{l}2,3+2,0+1,2+1,9+1,8+1,2+ \\
1,7+2,2+2,5+1,8=18,6\end{array}$ & 0,101 \\
\hline $\begin{array}{l}\text { Показа- } \\
\text { тель № } 2\end{array}$ & 1,7 & - & 1,7 & 2,0 & 0,9 & 2,1 & 3,6 & 1,4 & 2,4 & 1,7 & 1,5 & $\begin{array}{l}1,7+1,7+2,0+0,9+2,1+3,6+ \\
1,4+2,4+1,7+1,5=19\end{array}$ & 0,103 \\
\hline $\begin{array}{l}\text { Показа- } \\
\text { тель № } 3\end{array}$ & 2,0 & 2,3 & - & 2,3 & 2,2 & 1,1 & 2,1 & 2,9 & 1,4 & 2,2 & 1,9 & $\begin{array}{l}2,0+2,3+2,3+2,2+1,1+2,1+ \\
2,9+1,4+2,2+1,9=20,4\end{array}$ & 0,111 \\
\hline $\begin{array}{l}\text { Показа- } \\
\text { тель № } 4\end{array}$ & 2,8 & 2,0 & 1,7 & - & 1,3 & 1,2 & 1,3 & 1,6 & 1,1 & 2,7 & 2,2 & $\begin{array}{l}2,8+2,0+1,7+1,3+1,2+1,3+ \\
1,6+1,1+2,7+2,2=17,9\end{array}$ & 0,097 \\
\hline $\begin{array}{l}\text { Показа- } \\
\text { тель № } 5\end{array}$ & 2,1 & 3,1 & 1,8 & 2,7 & - & 2,6 & 2,4 & 1,4 & 1,8 & 1,3 & 2,3 & $\begin{array}{l}2,1+3,1+1,8+2,7+2,6+2,4+ \\
1,4+1,8+1,3+2,3=21,5\end{array}$ & 0,117 \\
\hline $\begin{array}{l}\text { Показа- } \\
\text { тель № } 6\end{array}$ & 2,2 & 1,9 & 2,9 & 2,0 & 1,4 & - & 1,2 & 1,1 & 1,5 & 2,1 & 1,5 & $\begin{array}{l}2,2+1,9+2,9+2,0+1,4+1,2+ \\
1,1+1,5+2,1+1,5=17,8\end{array}$ & 0,097 \\
\hline $\begin{array}{l}\text { Показа- } \\
\text { тель № } 7\end{array}$ & 2,8 & 0,4 & 1,9 & 2,7 & 1,6 & 2,8 & - & 2,2 & 2,1 & 2,0 & 2,2 & $\begin{array}{l}2,8+0,4+1,9+2,7+1,6+2,8+ \\
2,2+2,1+2,0+2,2=20,7\end{array}$ & 0,113 \\
\hline $\begin{array}{l}\text { Показа- } \\
\text { тель № } 8\end{array}$ & 3,3 & 2,6 & 1,1 & 2,4 & 2,6 & 2,9 & 1,8 & - & 2,0 & 2,6 & 2,5 & $\begin{array}{l}3,3+2,6+1,1+2,4+2,6+2,9+ \\
1,8+2,0+2,6+2,5=23,8\end{array}$ & 0,130 \\
\hline $\begin{array}{l}\text { Показа- } \\
\text { тель № } 9\end{array}$ & 1,8 & 1,6 & 2,6 & 2,9 & 2,2 & 2,5 & 1,9 & 2,1 & - & 2,1 & 2,5 & $\begin{array}{l}1,8+1,6+2,6+2,9+2,2+2,5+ \\
1,9+2,1+2,1+2,5=22,2\end{array}$ & 0,121 \\
\hline $\begin{array}{l}\text { Показа- } \\
\text { тель № } 10\end{array}$ & 1,5 & 2,3 & 1,8 & 1,3 & 2,7 & 1,9 & 2,0 & 1,4 & 1,9 & - & 2,9 & $\begin{array}{l}1,5+2,3+1,8+1,3+2,7+1,9+ \\
2,0+1,4+1,9+2,9=19,7\end{array}$ & 0,107 \\
\hline $\begin{array}{l}\text { Показа- } \\
\text { тель № } 11\end{array}$ & 2,2 & 2,5 & 2,1 & 1,8 & 1,7 & 2,5 & 1,9 & 1,5 & 1,5 & 1,1 & - & $\begin{array}{l}2,2+2,5+2,1+1,8+1,7+2,5+ \\
1,9+1,5+1,5+1,1=18,8\end{array}$ & 0,102 \\
\hline
\end{tabular}

Помимо этого, формируется таблица, куда заносятся значения стоимости каждого образца радиолокационной станции, из которых производится выбор, и допустимое значение стоимости радиолокационной станции (задается заказчиком) $C_{\text {треб. }}$, после чего формируется множество $C$ значений стоимости радиолокационных станций:

$$
C=\left\{C_{1}, C_{2}, \ldots, C_{M}\right\} .
$$

Исходя из этого на основании множества значений стоимости радиолокационных $C$ отбираются те радиолокационные станции, стоимость которых не превышает допустимого значения стоимости $C_{\text {треб. }}$. Далее из отобранных радиолокационных станций отбирается та радиолокационная станция, значение функции предпочтения $W_{j}$ которой максимально.

Таким образом, в статье предложен новый метод, позволяющий оценивать эффективность РЛС с помощью одного интегрального показателя. Дополнительные показатели позволят исключить неопределенность при выборе конкретных типов РЛС и выбирать радиолокационную 
станцию из всего множества доступных радиолокационных станций с учетом их стоимости по максимуму функции предпочтения.

\section{Список литературы}

[1] Бердышев В.П., Кордюков Р.Ю., Помазуев О.Н., Миронов А.М., Цыганов А.А., Стучилин А.И. Вероятностный подход к оценке разрешающих способностей РЛС по дальности и азимуту. Научный вестник оборонно-промышленного комплекса России, 2014, 2, 58-66. [Berdyshev V.P., Kordykov R.Y., Pomazuev O.N., Mironov A.M., Tsyganov A.A., Stuchilin A.I. Probabilistic approach to the assessment of the radar resolution capabilities in range and azimuth. Scientific Bulletin of the Russian Defense Industry Complex, 2014, 2, 58-66 (in Russian)]

[2] Цыганов А.А., Бердышев В.П., и др. Имитационная модель построения двумерных радиолокационных портретов воздушных объектов. Свидетельство о государственной регистрации программы для ЭВМ № 2018612397, заявка № 2017663663, дата пост. 26.21.2017 дата рег. 16.02.2018. ФГБУ ФИПС, 2018. [Tsyganov A.A, Berdyshev V.P. and others. Simulation model for the construktion of two-dimensional radar portraits of air assets. Certificate of state registration of a program for computer. FIIP, 2018 (in Russian)]

[3] Цыганов А.А., Конопацкий А.В. Оценка эффективности адаптивной по структуре системы селекции движущихся целей с цифровыми режекторными фильтрами. Материаль Всероссийской военно-научной конференции. Сборник материалов № 1. Тверь: ВА ВКО, 2018. [Tsyganov A.A., Konopacki A.V. Evaluation of the efficiency of a structurally adaptive selection system for moving targets with digital notch filters. Materials of the All-Russian Military Scientific Conference. Materials collection № 1. Tver, 2018. (in Russian)]

[4] Цыганов А.А., Бойкова А.В. Методика оценки затрат на эксплуатацию радиотехнического средства с учетом коэффициента дисконтирования. Журнал Сибирского федерального университета, Техника и технологии, 2018, 11(3), 347-357 [Tsyganov A.A., Boicova A.V. Methods of cost estimates for the operation on radio engineering means taking into account the discount factor. Journal of Siberian Federal University. Engineering \& Technologies, 2018, 11(3). 347-357 (in Russian)]

[5] Демидов Б. А. Теория иметоды военно-научных исследований вооружения ивоенной техники. Харьков: ВИРТА, 1990. С. 141 - 148 [Demidov B.A. Theory and methods of military-scientific research of weapons and military equipment. Kharkov: VIRTA, 1990. 141-148 (in Russian)]

[6] Буренок В.М., Ляпунов В.М., Мудров В.И. Теория и практика планирования и управления развитием вооружения. Под ред. А.М. Московского. М.: Вооружение. Политика. Конверсия, 2005, 95-100 [Burenok V.M., Lyapunov V.M., Mudrov V.I. Theory and practice of planning and managing the development of weapons. Under the editorship of A. Moscovsky. Moscow: Army. Policy. Conversion. 2005, 95-100 (in Russian)] 